Commun. math. Phys. 33, 243-251 (1973)

(C) by Springer-Verlag 1973

\title{
On the Domains of Generalized Operators
}

\author{
George Svetlichny \\ Departamento de Matemática, Pontifícia Universidade Católica, Rio de Janeiro, Brasil
}

Received March 12; in revised form June 16, 1973

\begin{abstract}
The domains of generalized operators $T: \Phi_{-} \rightarrow \Phi_{+}$on rigged Hilbert spaces $\Phi_{-} \subset H \subset \Phi_{+}$are investigated. We introduce an equivalence relation for operators with different domains. Arguments are given for taking $\Phi_{+}$to be the weak quasi-completion of $\Phi_{-}$and for $\Phi_{-}$to be Mackey quasi-complete. For domains of closed symmetric Hilbert space operators we give a representation for $\Phi_{+}$and provide certain elements in the equivalence class of the corresponding operator.
\end{abstract}

In a previous paper [1] we gave an introduction to a new systematic theory of sesquilinear forms on Hilbert space. We were generally concerned with forms that do not arise from associated operators with the particular aim of constructing a unified structure theory of self adjoint and symmetric operators and the formal Hamiltonians arising in quantum mechanical systems with infinitely many degrees of freedom. The heavy dose of extramathematical and physical reasoning in [1] does not allow for a satisfactory mathematical presentation, yet enough material was uncovered to begin the study of several purely mathematical problems. We here address ourselves to a circle of problems connected with the domains of definition of sesquilinear forms. For physical application the choice of the domain should not make any difference. Thus for a form given by a physical Hamiltonian in Fock space, it should not matter if the $n$-particle vectors are chosen from $\mathscr{D}$ or from $\mathscr{S}$ provided the form is defined on both. The forms are physically equivalent and we must reflect this fact by defining an appropriate mathematical equivalence. Furthermore, domain questions are related to regularity properties of the forms and these properties must be studied as part of the abstract theory.

This paper is self contained and no knowledge of [1] is necessary though here we present no motivations.

We make heavy use of the theory of topological vector spaces, but give references to all the results used. Our notations and conventions are the following:

$H$ is always a Hilbert space and the inner product $(\cdot, \cdot)$ is antilinear in the first variable; 
$\mathscr{D}$ is the space of $C^{\infty}$ functions of compact support;

$\mathscr{S}$ is Schwartz's space of rapidly decreasing functions;

For any given complex vector space $E$, we denote by $E^{*}$ the space of all antilinear functionals on $E$. Note that since no notion of continuity is yet defined, $E^{*}$ is the algebraic dual of $E$.

For any given vector space topology $\mathscr{T}$ on $E$ we write $E[\mathscr{T}]$ for the corresponding topological vector space;

The dual $E[\mathscr{T}]^{\prime}$ is always the set of antilinear functionals on $E[\mathscr{T}]$ continuous in the topology $\mathscr{T}$; this also applies to the distribution spaces. In particular $\mathscr{D}^{\prime}$ and $\mathscr{S}^{\prime}$ are the antilinear duals of $\mathscr{D}$ and $\mathscr{S}$ equipped with their usual topologies.

The indices $s, k, b$, and $\|\cdot\|$ stand respectively for the weak, Mackey, strong, and norm topology. (The notation follows [2]; note $s$ is short for schwach, not strong!)

If $\langle E, F\rangle$ is a duality then for the index $a=s, k$, or $b$ the corresponding topology on $E$ is denoted by $\mathscr{T}_{a}$, or $\mathscr{T}_{a}(F)$ depending on how much can be understood from the context; likewise, we write $E_{a}$ for $E\left[\mathscr{T}_{a}\right]$ when the duality is understood; by $A^{+}$.

If $A$ is a Hilbert space operator, its Hilbert space adjoint is denoted

By a rigged Hilbert space we mean a triple of complex vector spaces $\Phi_{-} \subset H \subset \Phi_{+}$where $H$ is a Hilbert space, $\Phi_{-}$is a dense subspace, and $\Phi_{+}$ is a space of antilinear functionals on $\Phi_{-}$. We denote the pairing between $\Phi_{-}$and $\Phi_{+}$by $\langle\cdot, \cdot\rangle$ or by $\langle\cdot, \cdot\rangle_{\Phi}$ when we need to make the rigging explicit.

By a generalized operator $T$ we mean a linear map $T: \Phi_{-} \rightarrow \Phi_{+}$. $T$ may be looked upon as a sesquilinear form on $\Phi_{-} ; f, g \leadsto\langle f, T g\rangle$. A sesquilinear form $S: f, g \leadsto S(f, g)$ on $\Phi_{-}$is not necessarily a generalized operator for if $\Phi_{+}$is strictly smaller than $\Phi_{-}^{*}$ some of the functionals $S(\cdot, g)$ may not belong to $\Phi_{+}$. We may always interpret $S$ as a linear map $S: \Phi_{-} \rightarrow \Phi_{-}^{*}$ but requiring its range to lie in $\Phi_{+}$is an imposition of a regularity condition.

Given a generalized operator $T$ we define the form $S(f, g)=\overline{\langle g, T f\rangle}$. If $S$ is also a generalized operator we call it the dual of $T$ and denote it by $T^{*}$. Thus when $T^{*}$ exists $\left\langle f, T^{*} g\right\rangle=\overline{\langle g, T f\rangle}$. When $T=T^{*}$ we say that $T$ is symmetric. When we wish to refer to both $T$ and $T^{*}$ without specifying which we shall write $T^{\S}$.

We henceforth always assume $T^{*}$ exists: This is a regularity assumption on $T$.

Theorem 1. The existence of $T^{*}$ is equivalent to the continuity of $T$ as a map $T: \Phi_{-s} \rightarrow \Phi_{+s}$.

Proof. By [2] (§ 20.4. (1)). 
Let now $\hat{\Phi}_{-} \subset H \subset \hat{\Phi}_{+}$be a different rigging of $H$. Assume $\hat{\Phi}_{-} \supset \Phi_{-}$, then we define the restriction map $\varrho: \hat{\Phi}_{+} \rightarrow \Phi_{-}^{*}$ by $\varrho \phi=\phi \mid \Phi_{-}$. If $T$ is a generalized operator in $\Phi$ and $\hat{T}$ a generalized operator in $\hat{\Phi}$, we say $\hat{T}$ is an extension of $T$ if and only if $\varrho \circ \hat{T}^{\S} \mid \Phi_{-}=T^{\S}$.

Theorem 2. Suppose $T$ has an extension $\hat{T}$, then this extension is unique if and only if $\varrho$ is injective.

Proof. Suppose $\varrho$ is injective and $\hat{T}_{1}$ and $\hat{T}_{2}$ are two extensions, then $\Delta=\hat{T}_{1}-\hat{T}_{2}$ satisfies $\varrho \circ \Delta^{\S} \mid \Phi_{-}=0$. From the injectivity of $\varrho$ follows $\Delta^{\S} \mid \Phi_{-}=0$. Hence for all $\hat{g} \in \hat{\Phi}_{-}$and $f \in \Phi_{-}$we have $\left\langle\hat{g}, \Delta^{\S} f\right\rangle_{\hat{\Phi}}=0$ and passing to the duals we get $\left\langle f, \Delta^{\S} \hat{g}\right\rangle_{\hat{\Phi}}=0$. Thus $\varrho \circ \Delta^{\S}=0$ and again using injectivity $\Delta^{\S}=0$.

Suppose now $\varrho$ is not injective and $\hat{T}$ is an extension. Then there is a nonzero element $\psi \in \hat{\Phi}_{+}$such that $\varrho \psi=0$. If by $|\psi\rangle\langle\psi|$ we denote the generalized operator in $\hat{\Phi}$ given by $|\psi\rangle\langle\psi| g=\psi \overline{\langle g, \psi\rangle}$ then setting $\hat{S}=\hat{T}+|\psi\rangle\langle\psi|$ we obtain a different extension of $T$.

Note that when $T$ is symmetric and has a unique extension $\hat{T}$ then $\hat{T}$ is also symmetric; for if it were not, $\hat{T}$ and $\hat{T}^{*}$ would be different extensions of $T$. If $T$ is symmetric and $\varrho$ is not injective then even requiring the extension to be symmetric cannot guarantee uniqueness for $|\psi\rangle\langle\psi|$ in the proof above is a symmetric form.

Let now $\Phi_{-}^{0} \subset H \subset \Phi_{+}^{0}$ be a rigging such that $\Phi_{-}^{0} \subset \Phi_{-}$. If $\varrho$ is the restriction map $\Phi_{+} \rightarrow \Phi_{-}^{0 *}$ and $T$ a generalized operator in $\Phi$ such that $\varrho \circ T^{\S}\left(\Phi_{-}^{0}\right) \subset \Phi_{+}^{0}$, then setting $T^{0}=\varrho \circ T \mid \Phi_{-}^{0}$ we obtain a generalized operator in $\Phi^{0}$. To show that $T^{0 *}$ exists we take $f, g \in \Phi_{-}^{0}$ and then we have $\left\langle f, T^{0} g\right\rangle_{\Phi^{0}}=\langle f, \varrho T g\rangle_{\Phi^{0}}=\langle f, T g\rangle_{\Phi}=\left\langle g, T^{*} f\right\rangle_{\Phi}=\left\langle g, \varrho T^{*} f\right\rangle_{\Phi^{0}}$. Since $\varrho \circ T^{*}\left(\Phi_{-}^{0}\right) \subset \Phi_{+}^{0}$ we see that $T^{0 *}=\varrho \circ T^{*} \mid \Phi_{-}^{0}$. In case $\varrho$ is injective $T$ is the unique extension of $T^{0}$.

By the rigging class of a generalized operator $T$ we mean the set of all generalized operators that can be obtained from $T$ by a finite sequence of extensions and/or restrictions as described above where in each step the corresponding restriction map $\varrho$ is required to be injective. A rigging class is clearly an equivalence class and it partly embodies the physical equivalence referred to in the introduction. As an example, the generalized operators of multiplication by the $\delta$ function $f(x) \leadsto \delta(x) f(0)$ defined either in $\mathscr{D} C L^{2} C \mathscr{D}^{\prime}$ or in $\mathscr{S} C L^{2} \subset \mathscr{S}^{\prime}$ belong to the same rigging class for $\varrho: \mathscr{S}^{\prime} \rightarrow \mathscr{D}^{\prime}$ is injective: It is to be especially noted that the various members of a rigging class generally have different riggings. In fact, it is precisely the necessity of collecting sesquilinear forms with different domains of definition into classes of equivalence that led to the notion of a rigging class.

An interesting but seemingly very difficult open problem is wheather two different generalized operators on a given rigged Hilbert space can 
belong to the same rigging class. That is, if by a sequence of restrictions and/or extensions we return to the initial rigged Hilbert space, do we also return to the same sesquilinear form? If we do, we can treat the form as an object independently of its many possible domains of definition; if not we are faced with very interesting structural questions. Even if one restricts the possibilities for $\Phi_{-}$and $\Phi_{+}$as we do in the next part of the paper, we still do not know the answer to this problem:

We now come to the problem of relating $\Phi_{-}$and $\Phi_{+}$. In concrete examples $\Phi_{+}$is the topological dual of $\Phi_{-}$in some topology. However, for a general theory we must effect such a construction from the only a priori material at hand which is the inner product in $\Phi_{\ldots}$. We are thus faced with the problem of extending a given pre-Hilbert space $(\Phi,(\cdot, \cdot))$ into a rigged Hilbert space $\Phi=\Phi_{-} \subset H \subset \Phi_{+}$. In such an extension we must reproduce the usual known situations and likewise obtain sufficiently many abstract properties for an effective theory. Starting from the duality $\langle\Phi, \Phi\rangle$ given by the inner product we can choose for $\Phi_{+}$at least any of the following possible candidates: (1) the space $\Phi_{s}^{0}$ of equivalence classes of Cauchy sequences of $\Phi_{s}$; (2) the sequential completion $\Phi_{s}^{\omega}$ of $\Phi_{s}$; (3) the strong double dual of $\Phi$, that is $\Phi_{b}^{\prime}$; (4) the quasi-completion $\Phi_{s}^{q}$ of $\Phi_{s}$. Each one of these can be interpreted as a space of anti-linear functionals on $\Phi$. For $\Phi_{b}^{\prime}$ this is so since $\Phi$ is in duality with itself; for the others it is true since each one is contained in the completion of $\Phi_{s}$ which by [2] (\$20.9. (2)) is equal to $\Phi^{*}$. Any one of these reproduces many of the familiar situations. However, for abstract reasons, we choose the fourth possibility. For the others, at least one of the following two theorems is either false or hasn't been established, and furthermore for a space $E[\mathscr{T}]$ to be weakly quasi-complete is equivalent to any of the following three very useful properties: (1) $E[\mathscr{T}]$ is semi-reflexive, that is $E_{b}^{\prime \prime}=E$; (2) in $E^{\prime}, \mathscr{T}_{b}=\mathscr{T}_{k} ;(3)$ in $E_{s}$ every bounded subset is relatively compact. See [2] (\$23.3) for reference.

Theorem 3. Let $\Phi_{-} \subset \hat{\Phi}_{-}$be two dense subspaces of the Hilbert space $H$; set $\Phi_{+}=\Phi_{-s}^{q}, \hat{\Phi}_{+}=\hat{\Phi}_{-s}^{q}$. Then the restriction map $\varrho: \hat{\Phi}_{+} \rightarrow \Phi_{-}^{*}$ maps all of $\hat{\Phi}_{+}$into $\Phi_{+}$.

Proof. $\varrho$ is continuous in the weak topologies given by the dualities $\left\langle\hat{\Phi}_{-}, \hat{\Phi}_{+}\right\rangle$and $\left\langle\Phi_{-}, \Phi_{-}^{*}\right\rangle$ for its dual exists being the inclusion map of $\Phi_{-}$into $\hat{\Phi}_{-} ;[2]\left(\S 20.4\right.$. (1)). Since $\Phi_{+s} \subset \Phi_{-s}^{*}$ is a quasi-closed subspace we have that $\Psi=\varrho^{-1}\left(\Phi_{+}\right) \subset \hat{\Phi}_{+s}$ is a quasi-closed subspace; [2] (\$ 23.1). Since $\hat{\Phi}_{+s}$ is quasi-complete, $\Psi\left[\mathscr{T}_{s}\left(\hat{\Phi}_{-}\right)\right]$is likewise quasi complete. On the other hand $\Psi \supset \varrho^{-1}(H) \supset H \supset \hat{\Phi}_{-}$, hence by definition of $\hat{\Phi}_{+}$ we have $\hat{\Phi}_{+}=\Psi$. Hence $\varrho^{-1}\left(\Phi_{+}\right)=\hat{\Phi}_{+}$but this means that $\varrho \hat{\Phi}_{+} \subset \Phi_{+}$. $\square$

If $\Phi_{\alpha}$ is a family of rigged Hilbert spaces we define the direct sum $\Phi=\oplus \Phi_{\alpha}$ by taking for $\Phi_{-}$that subspace of $\Pi \Phi_{\alpha-}$ whose elements have 
all but a finite number of components zero, and for $\Phi_{+}$the space $\Pi \Phi_{\alpha+}$. The pairing is $\langle f, \phi\rangle=\Sigma\left\langle f_{\alpha}, \phi_{\alpha}\right\rangle$, thus the Hilbert space in $\Phi_{-} \subset H \subset \Phi_{+}$ is the Hilbert space direct sum of the $H_{\alpha}$. If $\left(\Phi_{\alpha},(\cdot, \cdot)_{\alpha}\right)$ is a family of preHilbert spaces we define the direct sum $(\Phi,(\cdot, \cdot))$ by taking for $\Phi$ that subspace of $\Pi \Phi_{\alpha}$ whose elements have all but a finite number of components zero and setting $(f, g)=\Sigma\left(f_{\alpha}, g_{\alpha}\right)$ :

Theorem 4. Weak quasi-completions commute with direct sums; that is, the rigged Hilbert space associated to a direct sum of pre-Hilbert spaces is the direct sum of the rigged Hilbert spaces associated to each summand. In other words if $\left(\Phi_{\alpha},(\cdot, \cdot)_{\alpha}\right)$ is a family of pre-Hilbert spaces and $(\Phi,(\cdot, \cdot))$ is their direct sum, then $\Phi_{-s}^{q}=\Pi \Phi_{\alpha-s}^{q}$.

Proof. The product topology in $\Pi \Phi_{\alpha-s}^{q}$ is the topology induced by the weak topology on $\Phi_{-}^{*} ;[2](\S 22.5$. (3)). On the other hand since the product of quasi-complete spaces is quasi-complete [2] (\$23.1.(2)), we have that $\Phi_{+} \subset \Pi \Phi_{\alpha+}$. Let $F$ be a finite set of indices and set $\Phi_{F+}$ to be that subspace of $\Pi \Phi_{\alpha+}$ whose components for indices not belonging to $F$ are zero. $\Phi_{F+}$ is the weak quasi-completion of the similarly defined subspace $\Phi_{F-}$. This is clear since for each $\beta \in F, \Phi_{\{\beta\}+}$ is the weak quasicompletion of $\Phi_{\{\beta\}--}$. Now $\Phi_{F+}=\Sigma_{\beta \in F} \Phi_{\{\beta\}+}$ is quasi-complete [2] (§23.1. (2)) and since the quasi-completion of $\Phi_{F-}$ must contain each of the summands $\Phi_{\{\beta\}+}$, we see that $\Phi_{F+}$ is that quasi-completion. Hence we have $\Phi_{F+} \subset \Phi_{+}$. Let now $\phi \in \Pi \Phi_{\alpha+}$ and define $\phi_{F} \in \Pi \Phi_{\alpha+}$ by $\left(\phi_{F}\right)_{\alpha}$ being $\phi_{\alpha}$ if $\alpha \in F$ and zero if $\alpha \notin F$. Now $\left\{\phi_{F}\right\}$ is a bounded net for $\left\{\phi_{F}\right\}$ $\subset \Pi\left\{\phi_{\alpha}, 0\right\}$, and $\phi_{F} \rightarrow \phi$ in the weak topology of $\Phi_{-}^{*}$ since for each $f \in \Phi_{-},\left\langle f, \phi_{F}\right\rangle$ is eventually constant and equal to $\langle f, \phi\rangle$. Since each $\phi_{F} \in \Phi_{+}$and $\Phi_{+}$is weakly quasi complete, $\phi \in \Phi_{+}$hence $\Pi \Phi_{\alpha+} \subset \Phi_{+}$ proving the theorem.

For the remainder of the paper, unless otherwise stated, $\Phi_{+}$will always be taken to be the weak quasi-completion of $\Phi_{-}$. Such rigged Hilbert spaces we will call regular, and when regularity is not assumed we will call the rigged Hilbert space general. Some of the usual situations which regularity covers are $\mathscr{D} \subset L^{2} \subset \mathscr{D}^{\prime}, \mathscr{S} \subset L^{2} \subset \mathscr{S}^{\prime}, H \subset H \subset H$, and the nuclear rigged Hilbert spaces of Gelfand and Vilenkin [3]. In all of these cases $\Phi_{-}$is furnished with some original topology, and $\Phi_{+}$is the dual space. With this topology $\Phi_{-}$is reflexive in all of the above cases and hence $\Phi_{+}$is weakly quasi-complete [2] (§23.5. (3)); furthermore, in all of these cases every element of $\Phi_{+}$is a $\mathscr{T}_{s}\left(\Phi_{-}\right)$limit point of a sequence in $H$, hence $\Phi_{+}$is precisely the weak quasi-completion of $\Phi_{-}$. By Theorem 4 all direct sums of spaces of these types are likewise regular.

Given a regular rigged Hilbert space $\Phi_{-} \subset H \subset \Phi_{+}$, we can ask for which larger spaces $\hat{\Phi}_{-} \supset \Phi_{-}$is the restriction map $\varrho: \hat{\Phi}_{+} \rightarrow \Phi_{+}$bijective. That two different dense subspaces may have the same weak quasi- 
completion can be seen by taking for $\Phi_{-}$a dense hyperplane of the second category, then by applying the Banach-Steinhaus theorem one easily shows that $\Phi_{+}=H$. Since likewise $H_{+}=H$, we have an example. Our question is partially answered by the following theorem.

Theorem 5. Let $\Phi_{-} \subset H \subset \Phi_{+}$be a regular rigged Hilbert space, and let $\tilde{\Phi}_{-}=\tilde{\Phi}_{-k}$ be the completion of $\Phi_{-k}$. Then $\tilde{\Phi}_{-} \subset H$ and if $\Phi_{-} \subset \hat{\Phi}_{-} \subset \tilde{\Phi}_{-}$ then the restriction map $\varrho: \hat{\Phi}_{+} \rightarrow \Phi_{+}$is bijective.

Proof. By the Grothendieck construction of the completion [2] (§ 29.9. (2)) if $f \in \tilde{\Phi}_{\text {- then }} f$ is weakly continuous on the Mackey equicontinuous subsets of $\Phi_{+}$. Now $\Phi_{+}$being semi-reflexive, every weakly bounded subset is Mackey equicontinuous and weakly relatively compact. Since the $\mathscr{T}_{s}\left(\Phi_{-}\right)$closure $\bar{B}_{1}$ of the unit ball $B_{1}$ of $H$ in $\Phi_{+}$is weakly bounded, it is weakly compact, and $f$ being weakly continuous on $\bar{B}_{1}$ implies that $f$ is bounded on $\bar{B}_{1}$ and a fortiori on $B_{1}$. Hence $f \in H$ and so $\tilde{\Phi}_{-} \subset H$.

Let us assume now that $\hat{\Phi}_{-} \subset \tilde{\Phi}_{-}$. By [2] (\$23.8.(1)) the spaces $\Phi_{+}\left[\mathscr{T}_{s}\left(\Phi_{-}\right)\right]$and $\Phi_{+}\left[\mathscr{T}_{s}\left(\tilde{\Phi}_{-}\right)\right]$have the same bounded sets and these are furthermore all relatively compact. A fortiori $\Phi_{+}\left[\mathscr{T}_{s}\left(\hat{\Phi}_{-}\right)\right]$has the same bounded sets as the above two spaces and these likewise are all relatively compact. In particular this means that $\Phi_{+}\left[\mathscr{T}_{s}\left(\hat{\Phi}_{-}\right)\right]$is quasi-complete and so $\hat{\Phi}_{+} \subset \Phi_{+}$. Let now $B$ be a $\mathscr{T}_{s}\left(\Phi_{-}\right)$closed and bounded subset of $\Phi_{+}$, then $B$ is likewise $\mathscr{T}_{s}\left(\hat{\Phi}_{-}\right)$bounded and also a fortiori closed in $\hat{\Phi}_{+}$ in this stronger topology. Hence $B$ is $\mathscr{T}_{s}\left(\hat{\Phi}_{-}\right)$compact and consequently it is also compact and hence complete in the weaker topology $\mathscr{T}_{s}\left(\Phi_{-}\right)$. Thus $\hat{\Phi}_{+}$is $\mathscr{T}_{s}\left(\Phi_{-}\right)$quasi-complete which implies that $\hat{\Phi}_{+}=\Phi_{+}$.

We do not know if the converse of the theorem is true or not.

In the above theorem we can pick, in particular, $\hat{\Phi}_{-}=\Phi_{-k}^{q}$, the quasicompletion of $\Phi_{-k}$. That for the purposes of studying generalized operators we can always choose $\Phi_{\text {_ }}$ to be Mackey quasi-complete follows from the next theorem.

Theorem 6. Let $T$ be a generalized operator on a regular rigged Hilbert space $\Phi$. Let $\Phi^{\mu}$ be obtained by taking $\Phi_{-}^{\mu}=\Phi_{-k}^{q}$. Then $T$ has a unique extension $T^{\mu}$ to $\Phi^{\mu}$.

Proof. That the extension is unique, if it exists, follows from Theorems 2 and 5.

Since $T^{*}$ exists $T^{\S}: \Phi_{-s} \rightarrow \Phi_{+s}$ is continuous and hence a fortiori $T^{\S}: \Phi_{-k} \rightarrow \Phi_{+s}$ is continuous. Since $\Phi_{+s}$ is quasi-complete there exists a continuous extension $T^{\S \mu}: \Phi_{-k}^{\mu} \rightarrow \Phi_{+s}$; [2] (\$ 23.1. (4)). We need only show $T^{* \mu}=T^{\mu *}$. Now for $f \in \Phi_{-}$we have on $\Phi_{-}^{\mu},\left\langle\cdot, T^{*} f\right\rangle=\overline{\left\langle f, T^{\mu}(\cdot)\right\rangle}$ since both are Mackey continuous extensions of the same Mackey continuous functional on $\Phi_{-}$. Thus for all $g \in \Phi_{-}^{\mu}$ we have $\left\langle g, T^{*} f\right\rangle$ 
$=\overline{\left\langle f, T^{\mu} g\right\rangle}$. By the same reasoning we have again the following equality of functionals on $\Phi_{-}^{\mu}:\left\langle g, T^{* \mu}(\cdot)\right\rangle=\overline{\left\langle\cdot, T^{\mu} g\right\rangle}$. This establishes $T^{* \mu}$ $=T^{\mu *}$.

A rigged Hilbert space in which $\Phi_{-k}$ is quasi-complete we shall call Mackey regular. The above theorem states that we can always assume that a generalized operator is defined on a Mackey regular rigged Hilbert space provided we are only considering regular spaces. Combining [2] (§22.5. (4)) with [2] (§ 23.1. (2)) we see that a direct sum of Mackey regular spaces is likewise Mackey regular. This covers the usual riggings of Fock space.

We say that a generalized operator $T$ on a general rigged Hilbert space is bona fide if the range $\mathscr{R}\left(T^{\S}\right) \subset H$. Thus $T$ is a densely defined operator in Hilbert space the domain of whose Hilbert space adjoint contains $\Phi_{-}$. Not every member in the rigging class of a bona fide operator is necessarily bona fide. For example let $F$ be any $C^{\infty}$ real function on $R$ and consider in the two rigged Hilbert spaces $\mathscr{D} C L^{2} \subset \mathscr{D}^{\prime}$ and $\mathscr{S} \subset L^{2} \subset \mathscr{S}^{\prime}$ the generalized operator of multiplication by the function $T(x)=F(x) \cos \left(\int_{0}^{x} F(y) d y\right)$. Since $T(x)=\frac{d}{d x} \sin \left(\int_{0}^{x} F(y) d y\right)$ is a derivative of a bounded $C^{\infty}$ function, it is a tempered distribution and thus the multiplication is defined in both riggings giving rise to two symmetric operators in the same rigging class. For any such $F$ we have $T \mathscr{D} \subset \mathscr{D} \subset H$, hence $T$ is always bona fide in $\mathscr{D}$, however if $F$ grows sufficiently fast at infinity, for example $F(x)=e^{x^{2}}$, then $T \mathscr{S} \nsubseteq H$ and so on $\mathscr{S}, T$ is not bona fide.

The following theorem gives a characterization of being bona fide.

Theorem 7. Let $T$ be a generalized operator on a general rigged Hilbert space, then $T$ is bona fide if and only if $T^{\S}$ is continuous as a map from $\Phi_{-}$equipped with the $\|\cdot\|$ topology to $\Phi_{+s}$.

Proof. $T: \Phi_{-\|\cdot\|} \rightarrow \Phi_{+s}$ is continuous if and only if for every $g \in \Phi_{-}$ there is a constant $C_{g}$ such that for all $f \in \Phi_{-},\langle g, T f\rangle \mid \leqq C_{g}\|f\|$, but this is equivalent, with the same quantifications, to $\left|\left\langle T^{*} g, f\right\rangle\right| \leqq C_{g}\|f\|$, and this is equivalent to $T^{*} g \in H$ for all $g$. Hence the continuity of $T$ is equivalent to $\mathscr{R}\left(T^{*}\right) \subset H$ and interchanging $T$ and $T^{*}$ we complete the proof.

Theorem 8. Let $T$ be a bona fide generalized operator on a regular rigged Hilbert space, then $T^{\mu}$ is likewise bona fide.

Proof. Since $T$ is bona fide, $T^{\S}: \Phi_{-\|\cdot\|} \rightarrow \Phi_{+s}$ is continuous. If $B_{1}$ is the unit ball in $H$ then the $\mathscr{T}_{s}\left(\Phi_{-}\right)$closure of $T^{\S}\left(B_{1} \cap \Phi_{-}\right)$is $\mathscr{T}_{s}$ bounded and compact. By the Grothendieck completion construction and the quasi-completness of $\Phi_{+s}$, every element of $\Phi_{-}^{\mu}$ is weakly continuous 
on the bounded subsets of $\Phi_{+s}$. Hence for $f \in \Phi_{-}^{\mu},\left\langle f, T^{\S} g\right\rangle$ is bounded as $g$ varies in $B_{1} \cap \Phi_{-}$. Hence we have $\left|\left\langle\varrho T^{\mu \S} f, g\right\rangle\right| \leqq C_{f}\|g\|$ for $f \in \Phi_{-}^{\mu}$, $g \in \Phi_{-}$. But this means that $\varrho \circ T^{\mu \S}\left(\Phi_{-}^{\mu}\right) \subset H$ but since $\varrho$ is injective this means that $T^{\mu}$ is bona fide.

We now pass to the study of symmetric bona fide operators.

Theorem 9. Let $A$ be a closed symmetric Hilbert space operator with domain $\mathscr{D}(A)$, then $\mathscr{D}(A)_{+}$consists of all antilinear functionals $\phi$ on $\mathscr{D}(A)$ representable as $\langle f, \phi\rangle=((A+i) f,(A+i) l)$ where $l \in \mathscr{D}(A)$.

Proof. We first show that the space $\mathscr{F}$ of all such functionals is weakly quasi-complete. Let $\phi_{\alpha}$ be a weak bounded Cauchy net of such functionals. Then for all $f \in \mathscr{D}(A)$ we have $\left\langle f, \phi_{\alpha}\right\rangle=\left((A+i) f, k_{\alpha}\right)$ where $k_{\alpha} \in \mathscr{R}(A+i)$. Now $k_{\alpha}$ is therefore a $\mathscr{T}_{s}(\mathscr{R}(A+i))$ bounded Cauchy net in $\mathscr{R}(A+i)$, but since $\mathscr{R}(A+i)$ is closed and since a Hilbert space is weakly quasi-complete $k_{\alpha}$ converges weakly to an element $(A+i) l$ $\in \mathscr{R}(A+i)$. Hence $\left\langle f, \phi_{\alpha}\right\rangle \rightarrow((A+i) f,(A+i) l)$ establishing the quasicompleteness of $\mathscr{F}_{s}$.

Let now $B=(A+i)^{+}(A+i) ; B$ is a self adjoint operator and $B \geqq I$. Hence $J=B^{-1}$ exists and is bounded. Furthermore $J H=\mathscr{D}(B) \subset \mathscr{D}(A)$ and $\mathscr{D}(B)$ is a core for $A$. Let $h \in H$ then we have the functional in $\mathscr{F}$ given by $\langle f, \phi\rangle=((A+i) f,(A+i) J h)=(f, B J h)=(f, h)$. Hence $H \subset \mathscr{F}$.

Since $\mathscr{D}(B)$ is a core for $A$ there exists for any $l \in \mathscr{D}(A)$ a sequence $k_{n} \in \mathscr{D}(B)$ such that in the norm $k_{n} \rightarrow l, A k_{n} \rightarrow A l$. Hence given $\langle f, \phi\rangle$ $=((A+i) f,(A+i) l)$ we have $\langle f, \phi\rangle=\lim \left((A+i) f,(A+i) k_{n}\right)=\lim \left(f, B k_{n}\right)$. Thus every element of $\mathscr{F}$ is a weak limit point of a sequence of elements in $H$ which finally establishes $\mathscr{F}=\mathscr{D}(A)_{+}$.

In the above theorem the number $i$ can be replaced by any point in the regular set of $A$.

Theorem 10. Let $A$ be a closed symmetric Hilbert space operator, then $A^{\mu}=A$; that is, $\mathscr{D}(A)$ is Mackey regular.

Proof. Suppose $A^{\mu} \neq A$, then $A^{\mu}$ is a bona fide symmetric extension of $A$. Let $V$ be the Cayley transform of $A^{\mu}$; hence $V=\left(A^{\mu}-i\right)\left(A^{\mu}+i\right)^{-1}$, $A^{\mu}=i(1+V)(1-V)^{-1} . \quad$ Furthermore, $\quad \mathscr{D}(V)=\mathscr{R}(A+i) \oplus F$ where $F \perp \mathscr{R}(A+i), \mathscr{D}\left(A^{\mu}\right)=(1-V)(\mathscr{R}(A+i) \oplus F)$, and $\mathscr{D}(A)=(1-V) \mathscr{R}(A+i)$. If $f=(1-V) h \in \mathscr{D}\left(A^{\mu}\right)$ then an easy computation reveals $h=-\frac{i}{2}\left(A^{\mu}+i\right) f$. Now pick any $k \in F, k \neq 0$, and define the functional $\phi$ on $\mathscr{D}\left(A^{\mu}\right)$ by $\langle f, \phi\rangle=-\frac{i}{2}\left(\left(A^{\mu}+i\right) f, k\right)$. We have that $\langle(1-V) k, \phi\rangle=\|k\|^{2}$ hence $\phi \neq 0$, but $\phi \mid \mathscr{D}(A)=0$. Since $\mathscr{D}\left(A^{\mu}\right)$ is dense we have a sequence $g_{n} \in \mathscr{D}\left(A^{\mu}\right)$ such that $g_{n} \rightarrow k$ in the norm. Set $\left\langle f, \phi_{n}\right\rangle=-\frac{i}{2}\left(\left(A^{\mu}+i\right) f, g_{n}\right)$ 
$=-\frac{i}{2}\left(f,\left(A^{\mu}-i\right) g_{n}\right)$. Hence $\phi_{n} \in H$ and $\phi_{n} \rightarrow \phi$ weakly which means that $\phi \in \mathscr{D}\left(A^{\mu}\right)_{+}$. However $\phi \mid \mathscr{D}(A)=0$ contradicts the bijectivity of $\varrho: \mathscr{D}\left(A^{\mu}\right)_{+}$ $\rightarrow \mathscr{D}(A)_{+}$demonstrating that $A^{\mu}=A$.

Theorem 11. Let $A$ be a symmetric Hilbert space operator, then its closure $\bar{A}$ is in the same rigging class.

Proof. It is enough to show that $\varrho: \mathscr{D}(\bar{A})_{+} \rightarrow \mathscr{D}(A)_{+}$is injective. Let $\phi \in \mathscr{D}(\bar{A})_{+}$then by Theorem 9 there is a $k \in \mathscr{R}(\bar{A}+i)$ such that for $f \in \mathscr{D}(\bar{A})$, $\langle f, \phi\rangle=((\bar{A}+i) f, k)$. Since $\mathscr{R}(A+i)$ is dense in $\mathscr{R}(\bar{A}+i)$ this functional cannot vanish on $\mathscr{D}(A)$ unless $k=0$, that is $\phi=0$.

Theorem 12. Let $A$ be a self adjoint Hilbert space operator, and $\alpha$ its corresponding sesquilinear form, then $\alpha$ is in the rigging class of $A$.

Proof. We first show that $\varrho: \mathscr{D}(\alpha)_{+} \rightarrow \mathscr{D}(A)_{+}$is injective. Now $\mathscr{D}(\alpha)=\mathscr{D}\left(|A|^{\frac{1}{2}}\right)$ hence by the representation Theorem 9 we get for $f \in \mathscr{D}(\alpha)$, $\phi \in \mathscr{D}(\alpha)_{+}$that $\langle f, \phi\rangle=\left(\left(|A|^{\frac{1}{2}}+i\right) f,\left(|A|^{\frac{1}{2}}+i\right) l\right)$ for some $l \in \mathscr{D}(\alpha)$. Suppose this vanished on all $f \in \mathscr{D}(A)$, then we have $\left.0=\left(|A|^{\frac{1}{2}}+i\right) f,\left(|A|^{\frac{1}{2}}+i\right) l\right)$ $=((|A|+i) f, l)$ but this implies that $l=0$ since $B=|A|+I$ is a self adjoint operator, $B \geqq I$ and $\mathscr{D}(B)=\mathscr{D}(A)$, hence $B \mathscr{D}(A)=H$. Thus $\varrho$ is injective. To complete the proof we must show that $\alpha$ viewed as a linear transformation maps $\mathscr{D}(\alpha)$ into $\mathscr{D}(\alpha)_{+}$. In other words, given $g \in \mathscr{D}(\alpha)$ we must find an $h \in \mathscr{D}(\alpha)$ such that for all $f \in \mathscr{D}(\alpha)$ we have $\alpha(f, g)$ $=\left(\left(|A|^{\frac{1}{2}}+i\right) f,\left(|A|^{\frac{1}{2}}+i\right) h\right)$. By the spectral representation for $\alpha$ this means that $\alpha(f, g)=\int(1+|\lambda|) d\left(f, E_{\lambda} h\right)$ where $E$ is the spectral measure of $A$. Let now $h=\left(\int \frac{\lambda}{1+|\lambda|} d E_{\lambda}\right) g$, then $h \in \mathscr{D}(\alpha)$ since $g \in \mathscr{D}(\alpha)$ and the spectral integrand is bounded. And now $h$ satisfies the needed condition.

Note that $\alpha$ is not bona fide if $A$ is unbounded, for if it were it would be a proper symmetric extension of a self adjoint operator.

\section{References}

1. Svetlichny, G.: JMP 11, 3433 (70)

2. Köthe, G.: Topological Vector Spaces I, Berlin Heidelberg New York: Springer 1969

3. Gelfand, I. M., Ya. Vilenkin, N.: Generalized Functions, Vol. 4, New York: Academic Press 1969

G. Svetchlichny

Departamento de Matemática

Pontifícia Universidade Católica

Rua Marquês de São Vicente

Rio de Janeiro, Brasil 
